\title{
Photocount statistics in mesoscopic optics
}

\author{
S. Balog, P. Zakharov, and F. Scheffold* \\ Department of Physics, University of Fribourg, 1700 Fribourg, Switzerland \\ S.E. Skipetrov甘 \\ Laboratoire de Physique et Modélisation des Milieux Condensés/CNRS, \\ Maison des Magistères, Université Joseph Fourier, 38042 Grenoble, France
}

(Dated: July 21, 2018)

\begin{abstract}
We report the first observation of the impact of mesoscopic fluctuations on the photocount statistics of coherent light scattered in a random medium. Poisson photocount distribution of the incident light widens and gains additional asymmetry upon transmission through a suspension of small dielectric spheres. The effect is only appreciable when the average number $\bar{n}$ of photocounts becomes comparable or larger than the effective dimensionless conductance $g$ of the sample.
\end{abstract}

Since the Anderson's discovery that the propagation of a quantum particle can be blocked by disorder [1] and subsequent realization that this 'Anderson localization' can also take place for electromagnetic waves or photons (light) 2], the quest for observing it has become a breathtaking adventure $[3,4,5,6]$. Although the observation of microwave localization in quasi-one dimensional disordered samples 6 now seems to be accepted by the scientific community, the localization of visible light in strongly scattering, three-dimensional (3D) semiconductor powders [3] has been questioned [4]. The extensive subsequent work [5] has shown that the scattering strength of available disordered materials in the optical frequency range is not sufficient to clearly distinguish the impact of Anderson localization on the commonly measured quantities (such as the average transmission coefficient and the coherent backscattering cone) from the impact that would be produced by a weak absorption of light in diffuse regime. For this reason, well-established experimental results in the field of optical localization in 3D media are limited to 'precursors' of Anderson localization that can be observed under conditions of diffuse scattering: coherent backscattering and weak localization 7], long-range correlations [8, 9], and universal conductance fluctuations [9].

The mesoscopic optical phenomena that we cited above can be understood and discussed in the framework of classical physics as of 1905 , without appealing to quantum mechanics. The impact of quantum-mechanical effects on the coherent backscattering of light has been demonstrated in beautiful experiments on light scattering in cold atomic clouds 10. The quantum nature of scatterers (atoms) had to be taken into account to understand the low value of the coherent backscattering enhancement factor. Experimental studies revealing the quantum nature of light in multiple scattering have been reported only very recently [11], besides a considerable theoretical interest in this subject [12]. In particular, Kindermann et al. have predicted that disorder can substantially alter photon statistics of degenerate incoherent radiation. In the present Letter we report the first exper- imental observation of the impact of one of the precursors of Anderson localization - mesoscopic, long-range correlations - on the photon statistics of degenerate coherent light emitted by a conventional continuous laser. We interpret our results in the framework of the semiclassical theory of photoelectric detection 13], which appears to be sufficient under conditions of experiments reported here.

As first noted by Einstein 100 years ago 14], the quantum nature of light can be directly probed by the photoelectric effect. As the energy of the electromagnetic wave is quantized in portions $\hbar \omega$ (with $\hbar$ the Planck constant and $\omega$ the frequency of light), only an integer number $n$ of such quanta (photons) can be absorbed by a photoelectric effect-based detector during a given time interval $\tau$. Today's electronic equipment allows us to measure $n$ in a wide dynamic range and to determine the probability distribution of photocounts $P(n, \bar{n})$, where $\bar{n}$ is the average number of photocounts in the interval $\tau=\bar{n} / f$ and $f$ is the average photocount rate (number of counts per unit time). $P(n, \bar{n})$ carries fundamental information about interaction of light with the medium. In a 'random laser', for example, $P(n, \bar{n})$ can be used to characterize different regimes of lasing [15]. In a different domain of physics - mesoscopic electronics - the statistics of quasi-particle (electron) counts ('full counting statistics') in disordered conductors is also under active study [16].

In our experiment we measure the distribution of photocounts of laser light transmitted through an optically dense slab (see Fig. 1). The sample cell (thickness $L \approx 0.5 \mathrm{~mm}$ ) is filled with a charge stabilized aqueous colloidal dispersion of a commercial titanium dioxide powder (Warner Jenkinson Europe Ltd.), particle diameter $\approx 200-300 \mathrm{~nm}$, at an initial density of $18 \pm 1 \%$ per volume. To further increase the density we let the sealed suspension settle under gravity. Due to the electrostatic repulsion between the particles the sedimentation is asymptotically slowed down and an equilibrium layer of approx. $0.2-0.25 \mathrm{~mm}$ thickness (volume fraction ca. $35-40 \%$ ) is formed after about 10 hours. From diffusingwave spectroscopy measurements we have checked that 


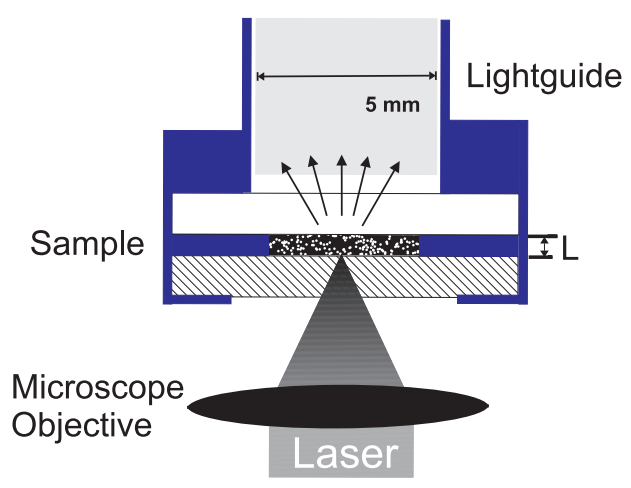

FIG. 1: Experimental setup: The incident laser beam is focused on the vertically oriented sample holder. The glass window directed towards the laser is highly absorbing while the opposite window is transparent. The windows hold a sealed liquid layer (thickness $L$ ) of colloidal titanium dioxide suspended in water. Light transmitted through the sample is collected by a light guide and recorded by a single photon detector (not shown).

the particles in this layer remain mobile and undergo Brownian motion. Following the approach described in reference [9] we estimate the transport mean free path at this density to $l^{*}=0.7 \pm 0.1 \mu \mathrm{m}$. Our sample is therefore deep in the multiple scattering regime: a typical transmitted photon experiences $\left(L / l^{*}\right)^{2} \sim 10^{5}$ elastic scattering events, whereas the coherent incident beam is destroyed after a distance $l^{*} \ll L$ and hence does not contribute to the measured signal. The glass window directed towards the laser is highly absorbing (transmission coefficient $\simeq 0.001$ ) in order to suppress multiple reflections [9] while the opposite one is transparent. A frequency doubled Nd:YV04 laser ('Verdi' from Coherent) operating at the wavelength $\lambda=532 \mathrm{~nm}$ illuminates the sample through a microscope objective that focuses the laser beam to a small spot (spot size $w \gtrsim 3 \mu \mathrm{m}$ ) on the sample surface. The light transmitted through the cell is collected by a light guide (core diameter $5 \mathrm{~mm}$ ), recorded by a single photon detector with short dead time, and processed by a digital photon counter (correlator.com, New Jersey, USA). The high temporal resolution (12.5 ns) of the latter assures that no more than one photon is arriving every time step for a typical photon count rate $f$ of $9 \mathrm{MHz}$. In a typical experiment the photon trace is recorded over one hour. From the recorded data we compute the probability distribution $P(n, \bar{n})$ and the time averaged correlation function of total transmission $C_{2}(t)=\langle T(0) T(t)\rangle / \bar{T}^{2}-1$ following standard procedures [18]. To suppress contributions from slow drifts the data is analyzed in thirty second intervals and subsequently averaged. Some representative results for different beam spot sizes are shown in Figs. 2 and 3 .

Because the detection process is of probabilistic nature, the detection of a photon is a random event and
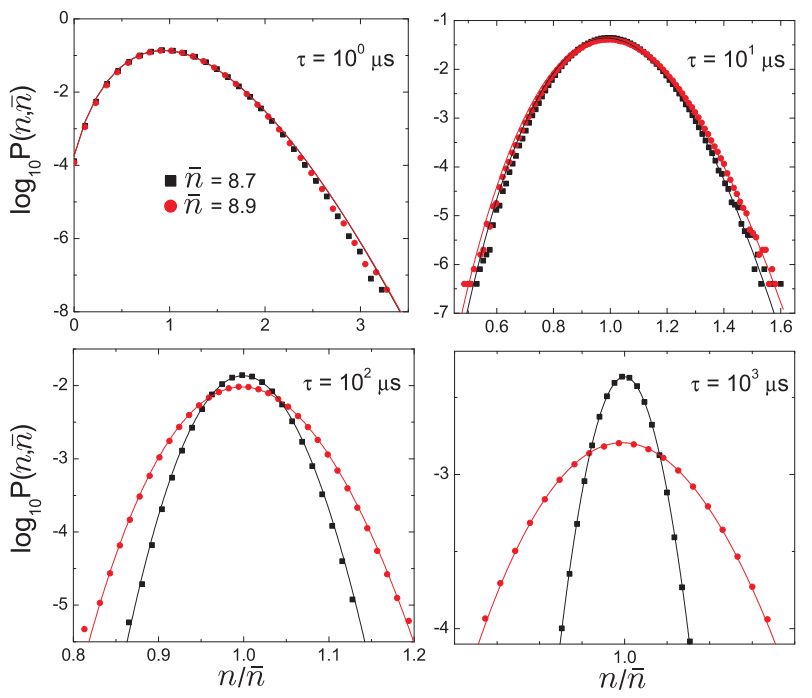

FIG. 2: Probability distributions of photon counts for a wide incident beam (black squares) and a focused beam (beam waist $w=3.4 \mu \mathrm{m}$, red circles) for four different sampling times $\tau$. The former distribution follows the Poisson law (black line), while the latter one is well described by the Fourier transform of our Eq. (3) (red line). Small deviations for the shortest $\tau$ can be explained by the finite detector dead time [17]. The number of data points for the two longest $\tau$ has been reduced to improve readability.

$P(n, \bar{n})$ is expected to be the Poisson probability distribution [13]. However, as follows from Fig. 2 this is only true when the incident laser beam is sufficiently wide. For a focused beam we observe that the distribution widens and becomes more asymmetric than one would expect for the Poisson distribution. This indicates that additional fluctuations exist in the latter case. These additional fluctuations are due to the random motion of scatterers. Since we collect all the transmitted intensity (total transmission measurements), and since the surface of our sample is much larger than the typical size $\sim \lambda$ of speckle spots, one could naively expect spatial speckle to average out, as it is indeed the case for large $w$. However, if $w$ is small and the scattering is sufficiently strong, coherent interferences of scattered light give rise to weak but longrange correlation of distant speckle spots, which acquire a synchronous component in their fluctuations. This results in enhanced fluctuations of the total transmitted signal [9].

The enhanced fluctuations of the total transmission $T$ can be studied assuming that light is a classical wave described by Maxwell equations [19, 20]. As long as localization effects are weak, the statistical distribution $P_{T}(T)$ of $T$ appears to be very close to Gaussian (because the many speckle spots contributing to $T$ are only weakly correlated and the central limit theorem applies), but with enhanced second and non-zero third central moments $M_{T}^{(2)}$ and $M_{T}^{(3)}$, where $M_{T}^{(k)}=\left\langle(T-\bar{T})^{k}\right\rangle / \bar{T}^{k}$. Therefore, the characteristic function of $T$ can be ap- 


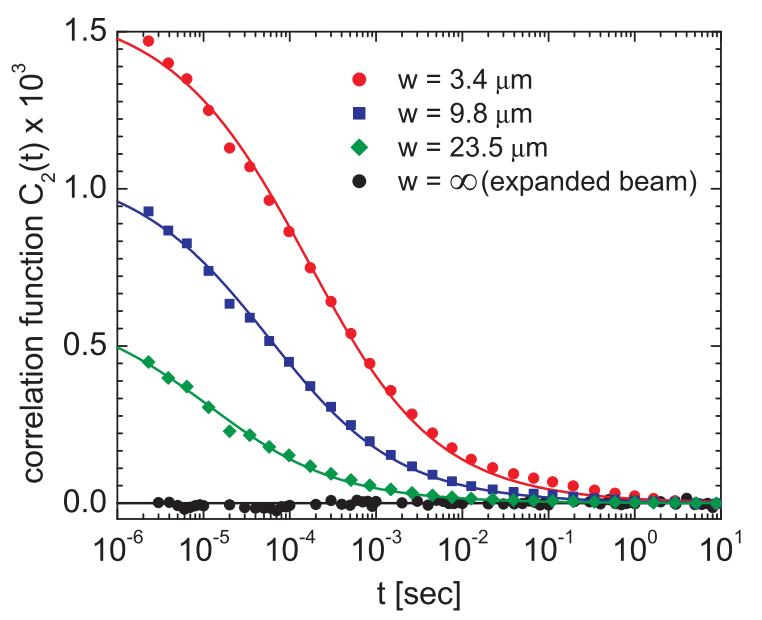

FIG. 3: Correlation function of total transmission $T$ for different beam spot sizes $w$ (distance between $1 / e$ intensity values of a focused Gaussian beam). Lines are fits to the data.

proximated by

$$
\chi_{T}(q)=\exp \left(i \bar{T} q-\frac{1}{2} \bar{T}^{2} M_{T}^{(2)} q^{2}-\frac{i}{6} \bar{T}^{3} M_{T}^{(3)} q^{3}\right)
$$

When the measurement of $T$ is not instantaneous but involves time integration, the second moment is given by 13.

$$
M_{T}^{(2)}=\frac{2}{\tau} \int_{0}^{\tau}\left(1-\frac{t}{\tau}\right) C_{2}(t) d t
$$

The third moment $M_{T}^{(3)}$ of the distribution of $T$ can be shown to be proportional to the square of the second one: $M_{T}^{(3)}=\alpha M_{T}^{(2) 2}$, where the proportionality constant $\alpha=16 / 5$ for a wide $(w \gg L)$ Gaussian beam [20]. The limit of wide beam has also been studied in the previous correlation experiments [9] and the corresponding correlation function $C_{2}(t)$ has been analyzed theoretically 21]. In our experiments, on the contrary, the beam width $w$ is much smaller than the thickness $L$ of the sample (typically, $w / L \sim 10^{-2}$ ). In this situation, by performing calculations similar to that of Ref. 21] we find $C_{2}(t)=(2 / 3 g) \exp \left(3 t / 4 t_{0}\right)\left[1-\Phi\left(\sqrt{3 t / 4 t_{0}}\right)\right]$, where $\Phi$ is the error function. Leaving the discussion of the microscopic expressions for $g$ and $t_{0}$ for a future publication 22], we just note here that $g$ scales roughly as $1 / w$ and hence the magnitude of the total transmission fluctuations $(\sim 1 / g)$ can be varied by adjusting the beam spot size $w$. By analogy with the case of disordered waveguide [6], we will further term $g$ the 'effective' dimensionless conductance. The above expression for $C_{2}(t)$ with $g \sim 10^{3}$ and $t_{0} \sim 10^{-5}-10^{-4}$ s provides a good fit to our measurements (see Fig. 3).

According to the famous Mandel's formula [13], the statistical distribution of photocounts $P(n, \bar{n})$ can be obtained by averaging the Poisson distribution $P_{\text {Poisson }}(n, \bar{n}=\eta T)$ over the distribution $P_{T}(T)$ of the total transmission $T$, with $\eta$ the quantum efficiency of the photodetector. The Fourier transform of the Mandel's formula with respect to $n$ yields a relation between the characteristic functions $\chi_{n}(q)$ and $\chi_{T}(q)$ :

$$
\begin{aligned}
\chi_{n}(q) & =\chi_{T}\left[i \bar{n}\left(1-e^{i q}\right) / \bar{T}\right] \\
& \simeq \exp \left(i \bar{n} q-\frac{1}{2} \bar{n}^{2} M_{n}^{(2)} q^{2}-\frac{i}{6} \bar{n}^{3} M_{n}^{(3)} q^{3}\right)
\end{aligned}
$$

where the second line is obtained by expanding the first one in power series in $q$, which is justified for large $\bar{n}$. The second and the third central moments of $n$ in Eq. (3) are

$$
\begin{aligned}
& M_{n}^{(2)}=\frac{1}{\bar{n}}+M_{T}^{(2)} \\
& M_{n}^{(3)}=\frac{1}{\bar{n}^{2}}+M_{T}^{(3)}+3 \frac{M_{T}^{(2)}}{\bar{n}}
\end{aligned}
$$

The Fano factor $\left.F=\left(\left\langle n^{2}\right\rangle-\bar{n}^{2}\right) / \bar{n}=1+\bar{n} M_{T}^{(2)}\right\rangle$ 1 , which indicates photon bunching. The photocount distribution $P(n, \bar{n})$ obtained by the Fourier transform of Eq. (3) describes our measurements very well (see Fig. (4), when we use the fits to the correlation function $C_{2}(t)$ obtained independently (Fig. 3) to determine $M_{T}^{(2)}$ and $M_{T}^{(3)}$.

The first terms on the right-hand sides of Eqs. (44) and (5) correspond to the results expected for Poisson distribution of photocounts. As we show in Fig. 4 these results are recovered for small $\bar{n} \ll g$, when the photocount distribution is dominated by the shot noise due to the discreteness of $n$ and is not sensitive to the randomness of the scattering medium. Deviations from the Poisson-like behavior start to become important when $\bar{n}$ becomes comparable to the effective dimensionless conductance $g$. The long-range character of the correlation function $C_{2}(t) \sim 1 / \sqrt{t}$ is responsible for new scaling laws $M_{n}^{(2)} \sim 1 / \sqrt{\bar{n}}$ and $M_{n}^{(3)} \sim 1 / \bar{n}$ in the limit of large $\bar{n}$. This behavior is well confirmed by our measurements: as can be seen in Fig. 4 the data points indeed follow the $1 / \sqrt{\bar{n}}$ and $1 / \bar{n}$ asymptotes shown by dashed lines 23]. Mesoscopic fluctuations of the total transmission $T$ due to the random motion of scatterers in the disordered sample become dominant in this regime, whereas the shot noise is negligible. We see therefore that the transition between small- and large- $\bar{n}$ regimes in $P(n, \bar{n})$ is governed by localization effects, the strength of the latter being measured by the dimensionless conductance $g$.

In conclusion, mesoscopic fluctuations of coherent light transmission through a random medium produce measurable deviations of photocount distribution $P(n, \bar{n})$ from Poisson law, provided that the average number of photocounts $\bar{n}$ is comparable or larger than the effective dimensionless conductance $g$ of the random sample. This 

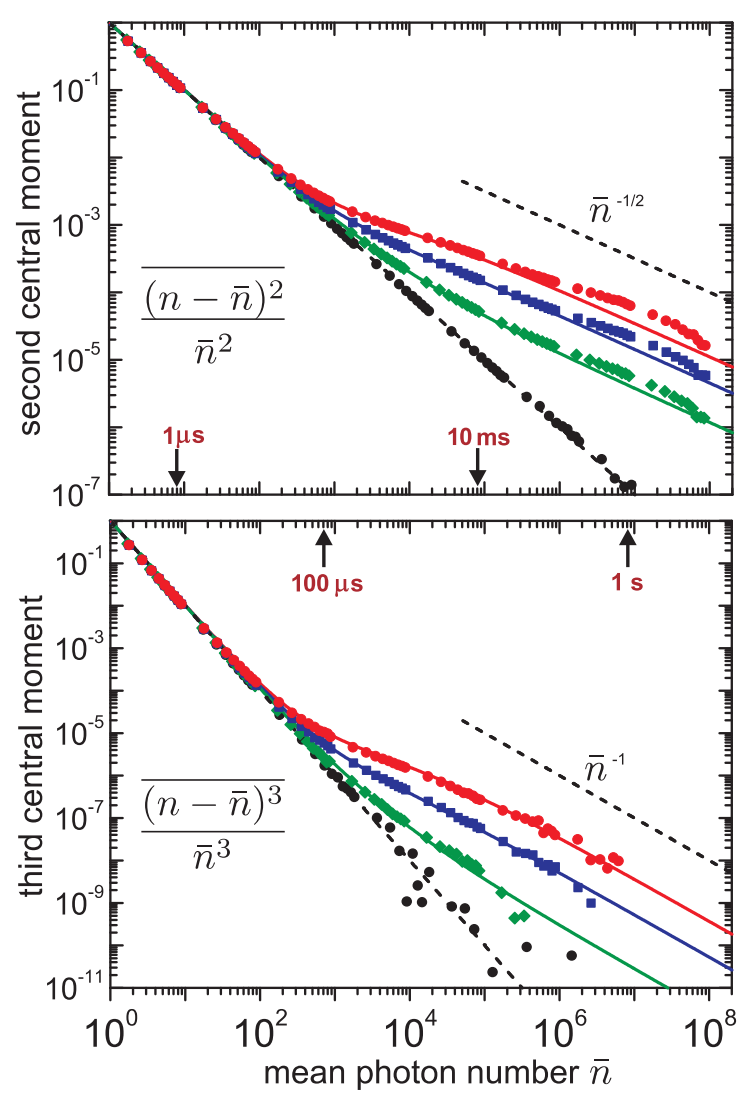

FIG. 4: Second and third central moments of the photocount distribution $P(n, \bar{n})$ for the same beam spot sizes as in Fig. 3 Lines are theoretical results (4) and (5). The third moment plot is a fit to the data with $\alpha=3.02,2.59$ and 2.00 (for curves from top to bottom).

provides a new tool for studying mesoscopic phenomena in random media, a tool that should be particularly valuable in the search for Anderson localization of light. An interesting continuation of this work would be to analyze $P(n, \bar{n})$ for incident light in thermal or in non-classical (Fock, squeezed, etc.) states.

This work was financially supported by the Swiss National Science Foundation (project No. 200021-101620). S.E.S. acknowledges financial support of INTAS through a Young Scientist Fellowship at the early stage of this work.

* Frank.Scheffold@unifr.ch

† Sergey.Skipetrov@grenoble.cnrs.fr

[1] P.W. Anderson, Phys. Rev. 109, 1492 (1958).

[2] S. John, Phys. Rev. Lett. 53, 2169 (1984); P.W. Anderson, Phil. Mag. B 52, 505 (1985); S. John, Phys. Today 44(5), 32 (1991).

[3] D.S. Wiersma, P. Bartolini, A. Lagendijk, and R. Righini, Nature 390, 671 (1997).
[4] Comment on Ref. [3]: F. Scheffold, R. Lenke, R. Tweer, and G. Maret, Nature 398, 206 (1999); Reply to comment: D.S. Wiersma, J. Gómez Rivas, P. Bartolini, A. Lagendijk, and R. Righini Nature 398, 207 (1999).

[5] J. Gómez Rivas, R. Sprik, C.M. Soukoulis, K. Busch, and A. Lagendijk, Europhys. Lett. 48, 22 (1999); F.J.P. Schuurmans, M. Megens, D. Vanmaekelbergh, and A.Lagendijk, Phys. Rev. Lett. 83, 2183 (1999); J. Gómez Rivas, R. Sprik, A. Lagendijk, L.D. Noordam, and C.W. Rella, Phys. Rev. E 63, 046613 (2001).

[6] A.A. Chabanov, M. Stoytchev, A.Z. Genack, Nature 404, 850 (2000).

[7] M.P. van Albada and A. Lagendijk, Phys. Rev. Lett. 55, 2692 (1985); P.-E. Wolf and G. Maret, Phys. Rev. Lett. 55, 2696 (1985); E. Akkermans, P. E. Wolf, and R. Maynard, Phys. Rev. Lett. 56, 1471 (1986).

[8] M.P. Van Albada, J.F. de Boer, and A. Lagendijk, Phys. Rev. Lett. 64, 2787 (1990)

[9] F. Scheffold and G. Maret, Phys. Rev. Lett. 81, 5800 (1998); F.Scheffold, W.Härtl, G. Maret, and E. Matijević, Phys. Rev. B 56, 10942 (1997).

[10] G. Labeyrie, F. de Tomasi, J.-C. Bernard, C.A. Müller, Ch. Miniatura and R. Kaiser, Phys. Rev. Lett. 83, 5266 (1999); T. Jonckheere, C.A. Müller, R. Kaiser, Ch. Miniatura and D. Delande, ibid. 85, 4269 (2000).

[11] P. Lodahl and A. Lagendijk, Phys. Rev. Lett. 94, 153905 (2005).

[12] M. Patra and C. W. J. Beenakker, Phys. Rev. A 60, 4059 (1999); ibid. 61, 063805 (2000); C. W. J. Beenakker, M. Patra, and P. W. Brouwer, ibid. 61051801 (2000); M. Kindermann, Yu. V. Nazarov, and C.W.J. Beenakker, Phys. Rev. Lett. 88, 063601 (2002); P. Lodahl, A.P. Mosk, and A. Lagendijk, Phys. Rev. Lett. 95, 173901 (2005).

[13] L. Mandel and E. Wolf, Optical Coherence and Quantum Optics (Cambridge Univ. Press, 1995).

[14] A. Einstein, Ann. Phys. 17, 132 (1905).

[15] H. Cao, Y. Ling, J.Y Xu, C.Q. Cao, P. Kumar, Phys. Rev. Lett. 86, 4524 (2001); M. Patra, Phys. Rev. A 65, 043809 (2002); L. Florescu and Sajeev John, Phys. Rev. Lett. 93, 013602 (2004).

[16] C.W.J. Beenakker and M. Büttiker, Phys. Rev. B 46, R1889 (1992); L.S. Levitov, H. Lee, and G.B. Lesovik, J. Math. Phys 37, 4845 (1996); D.A. Bagrets, Phys. Rev. Lett. 93, 236803 (2004).

[17] F.A. Johnson, R. Jones, T.P. McLean, and E.R. Pike, Phys. Rev. Lett. 16, 589 (1966).

[18] K. Schätzel, Quantum Opt. 2, 287-305 (1990).

[19] J.F. de Boer, M. C. W. van Rossum, M. P. van Albada, Th. M. Nieuwenhuizen and A. Lagendijk, Phys. Rev. Lett 73, 2567 (1994).

[20] M.C.W. van Rossum, J.F. de Boer, and Th.M. Nieuwenhuizen, Phys. Rev. E 52, 2053 (1995); M.C.W. van Rossum and Th.M. Nieuwenhuizen, Rev. Mod. Phys. 71, 313 (1999).

[21] R. Berkovits and S. Feng, Phys. Rep. 238, 135 (1994).

[22] B. Sandor, S.E. Skipetrov and F. Scheffold, in preparation.

[23] We attribute the slight curvature of experimental curves in Fig. 4 to slow fluctuations and drifts of the laser intensity on a time scale of the order of minutes. Though already extremely small in our experiments, these effects cannot be completely suppressed. 